MKG-Chirurg 2012 · 5:182-184

DOI 10.1007/s12285-012-0301-3

Online publiziert: 8. September 2012

(c) Springer-Verlag 2012

B. Al-Nawas ${ }^{1} \cdot$ H.-P. Ulrich ${ }^{2}$

${ }^{1}$ Klinik für Mund-, Kiefer- und Gesichtschirurgie - Plastische Operationen, Universitätsmedizin Mainz

${ }^{2}$ MKG-Chirurgie LindenArcaden, Lübeck

\title{
Hygiene in der MKG-Chirurgie
}

\section{Immer mehr, immer aufwendiger, immer erfolgreicher?}

Standpunkt, der sicherlich auch umgekehrt zu formulieren wäre.

fektion, die kenhaus, erworben wurde, hat die Politik den Kampf angesagt. Während man diesen Begriff früher nur auf die stationären Abläufe im Krankenhaus bezog, wird heute auch das ambulante Gesundheitswesen miteinbezogen. Für uns als MKG-Chirurgen spielt das politische Ziel, die Rate der postoperativen Wundinfektionen $\mathrm{zu}$ senken, eine große Rolle. Laut NIDEP-1Studie 1994 gehören die postoperativen Wundinfektionen zu den dritthäufigsten nosokomialen Infektionen. Sie führen zu einer deutlichen Erhöhung der stationären Verweildauer und der Behandlungskosten. Für den ambulanten Sektor liegen diesbezüglich kaum valide Daten vor. Gerne werden Schätzungen aus Großbritannien zitiert, die zusätzliche Behandlungskosten von 930 Mio. GBP pro Jahr aufgrund nosokomialer Infektionen kalkulieren (Gesundheitsberichterstattung des Bundes 2002). Auch wenn diese Daten, die auf Schätzungen beruhen, kritisch zu hinterfragen sind, so bleibt die „medienwirksame“ Reduktion der Rate nosokomialer Infektionen erklärtes politisches Ziel.

\section{\) Die Reduktion der Rate nosokomialer Infektionen ist erklärtes politisches Ziel}

Das Stichwort aus der sektorübergreifenden Versorgung lautet: „Die Operation im ambulanten Bereich darf nicht risikoreicher sein als im stationären Bereich." Ein
Bedeutsam ist, dass die Rate der postoperativen Wundinfektionen („surgical site infections“) von der Kontamination des Operationsfelds abhängt. Die klassische enorale MKG-Chirurgie findet, gemäß CDC-Kriterien, im „kontaminierten“ oder „sauber-kontaminierten“ Operationsfeld statt. Kriterien von Operationen, die im „sauberen“ Operationsfeld stattfinden, können also nicht unmittelbar auf unser Fach übertragen werden. Die Rolle endogener Faktoren bei enoralen Operationen ist bisher nicht geklärt.

Vor diesem Hintergrund ist die aktuelle Revision des Infektionsschutzgesetzes zu sehen. Auf der Basis des brisanten $\$ 23$ wird von Leitern von Einrichtungen für ambulantes Operieren erwartet, dass nosokomiale Infektionen und das Auftreten von Krankheitserregern mit Multiresistenzen fortlaufend aufgezeichnet und bede wird, darauf hinzuweisen, dass in einer ambulanten MKG-chirurgischen Praxis nur sehr selten ambulantes Operieren erfolgt, sondern meist klassische Eingriffe durchgeführt werden (Bundesgesundheitsblatt 1997). Die Erfahrung aus Begehungen zeigt, dass hier die Begriffsdefinitionen von den Verantwortlichen selten korrekt zugeordnet werden. Surveillance, also die Überwachung der postoperativen Infektionen im Sinne der Ergebnisqualität, wird als zentraler Aspekt angesehen. So wurde unlängst das AQUA-Institut vom Bundesgesundheitsministerium beauftragt, fachbewertet werden. Auch wenn man nicht mü- zogene Indikatoroperationen für Operationen festzulegen, die sowohl ambulant als auch stationär in relevanter Zahl durchgeführt werden. Bisher gibt es hierzu keinen Konsens. Unumgänglich wird für unser Fach die eigene Definition einer fachspezifischen sinnvollen Indikatoroperation. Damit sollte unter Berücksichtigung der politischen und gesetzlichen Vorgaben die Surveillance gesichert und die Ergebnisqualität dokumentiert werden.

Die zweite „Großbaustelle“ stellt die Aufbereitung von Medizinprodukten dar. Hier haben die meisten Kollegen vorbildliche Konzepte etabliert. Ähnlich wie bei den nosokomialen Infektionen spielen auch hier die Ausbildung und Schulung des Personals eine zentrale Rolle. Die Deutsche Gesellschaft für Sterilgutversorgung bietet dazu - man kann vermuten aus Eigeninteresse - ein mittlerweile 120-stündiges Curriculum an, das für einen Mitarbeitenden aus einem Medizinfachberuf nicht geeignet sein kann, sondern für fachfremdes Personal konzipiert sein mag.

\section{\) Ausbildung und \\ Schulung des Personals spielen eine zentrale Rolle}

In den Diskussionen im Arbeitskreis Krankenhaus- und Praxishygiene mit dem Vertreter des Robert-Koch-Instituts wurde deutlich, dass es Aufgabe der Fachgesellschaften ist, die Besonderheiten der Aufbereitung in ihrem Fach zu vermitteln. Unter dieser Idee entstand der Kurs zur 
Hier steht eine Anzeige.

黛 Springer 


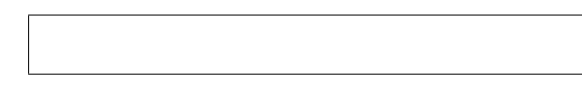

Aufbereitung von Medizinprodukten, der sich speziell an die Belange unseres Faches richtet und erfolgreich im Rahmen unserer Tagungen abgehalten wurde. Das große Echo auf diese Veranstaltung zeigt, wie ernst unsere Mitglieder dieses Thema nehmen. Damit wird deutlich, dass die MKG-Chirurgie bereits hohe Standards im Bereich Infektionsprävention, Hygiene und Instrumentenaufbereitung erreicht hat. Für die Zukunft müssen wir in einen engen Dialog mit den Beteiligten in Politik und Hygiene sowie den Kostenträger treten, um die Besonderheiten und den Standard in unserem Fach zu erläutern.

Fassen Sie dieses Heft als unseren Beitrag zu diesem wichtigen Thema auf; wir freuen uns auf Ihre Anregungen.

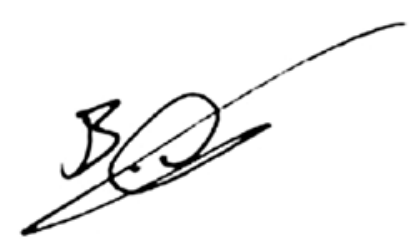

Prof. Dr. Dr. Bilal Al-Nawas

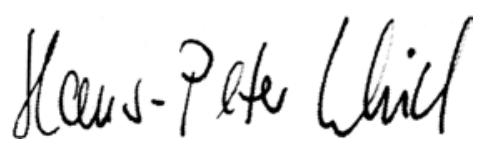

Dr. Dr. Hans-Peter Ulrich

\section{Korrespondenzadressen}

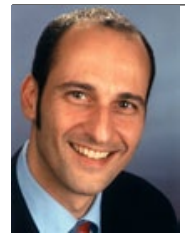

Prof. Dr. Dr. B. Al-Nawas

Klinik für Mund-, Kiefer- und Gesichtschirurgie - Plastische Operationen,

Universitätsmedizin Mainz

Augustusplatz 2, 55131 Mainz

bilal.al-nawas@

unimedizin-mainz.de

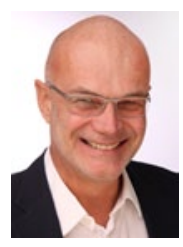

Dr. Dr. H.-P. Ulrich

MKG-Chirurgie LindenArcaden

Fackenburger Allee 1,

23554 Lübeck

hans-peter.ulrich@mkg-

chirurgie.de

\section{Leitthemenübersicht}

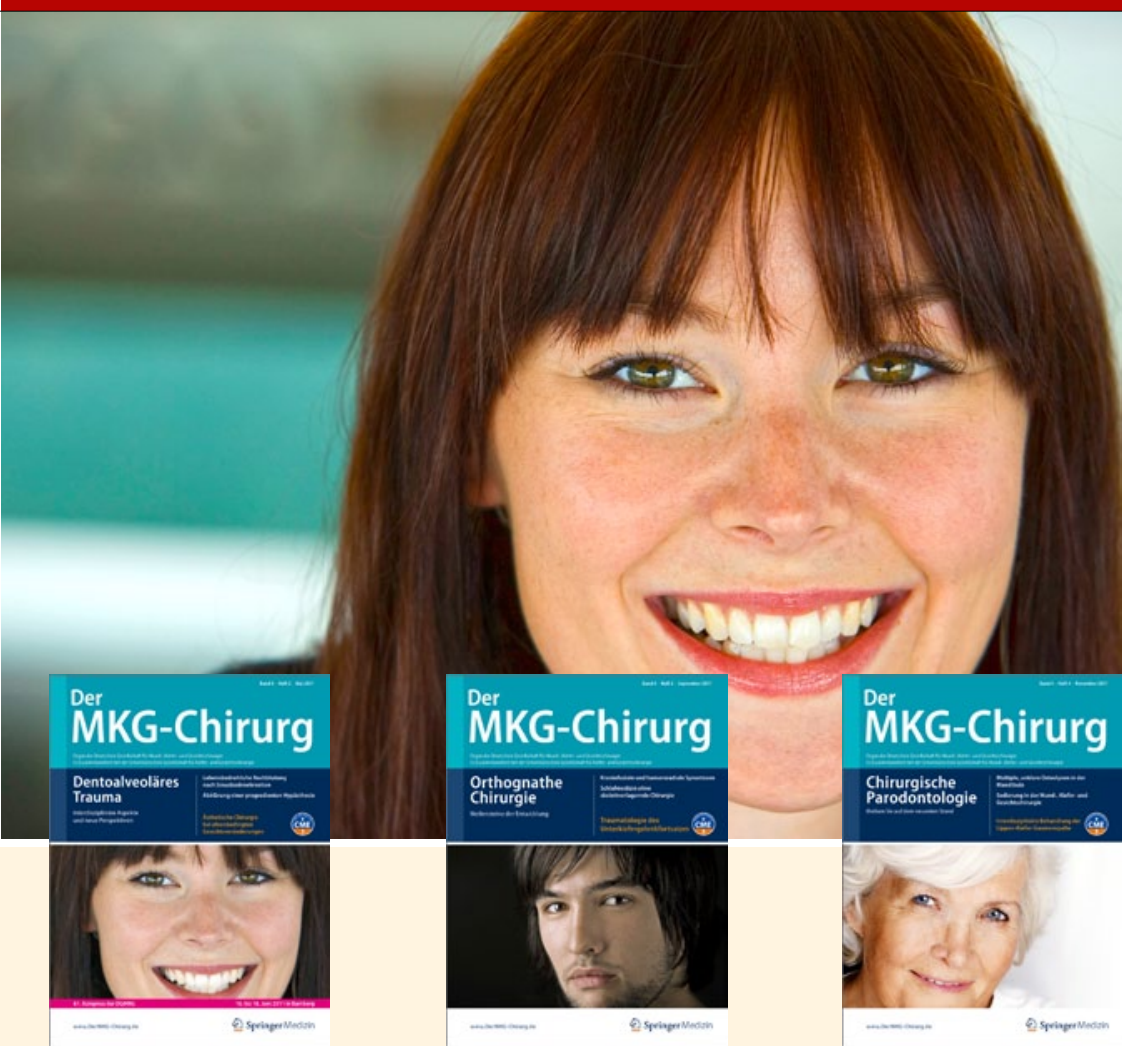

Der MKG-Chirurg bietet Ihnen umfassende und aktuelle Beiträge zu interessanten Themenschwerpunkten aus allen Bereichen der Mund-, Kiefer- und Gesichtschirurgie.

Möchten Sie ein bereits erschienenes Heft nachbestellen?

Einzelne Ausgaben können Sie direkt bei unserem Kundenservice zum Preis von je EUR 48,- zzgl. Versandkosten beziehen:

\section{Überblick 2012}

$\begin{array}{ll}\text { 1/12 } & \text { Gesicht und Identität } \\ \text { 2/12 } & \text { Sedierungen } \\ \text { 3/12 } & \text { Hygiene } \\ \text { 4/12 } & \text { Craniofaziale Chirurgie }\end{array}$

So erreichen Sie unseren Kundenservice:

Springer Customer Service Center GmbH Kundenservice Zeitschriften Haberstr. 7, 69126 Heidelberg

Tel.: +496221 345-4303

Fax: +49 6221 345-4229

E-Mail: leserservice@springer.com

www.DerMKG-Chirurg.de

\section{Vorschau 2013}

1/13 Fehlbildungen

2/13 Osteomyelitis und ihre Sonderformen

3/13 Rekonstruktive Chirurgie

4/13 Gutachten

(Änderungen vorbehalten) 